\title{
THE EXTENSION OF A HOMEOMORPHISM DEFINED ON THE BOUNDARY OF A 2-MANIFOLD
}

\author{
J. W. T. YOUNGS ${ }^{1}$
}

1. Introduction. Suppose that $M$ and $\mathfrak{M}$ are homeomorphic 2-manifolds with boundaries $B$ and $\mathfrak{B}$, respectively، Then $B(\mathfrak{B})$ is the union of a collection $J_{1}, \cdots, J_{n}\left(\Im_{1}, \cdots, \Im_{n}\right), n>0$, of Jordan curves which are disjoint in pairs. Suppose $h$ is a homeomorphism from $B$ onto $\mathfrak{B}$. (It may be assumed that $h\left(J_{i}\right)=\Im_{i}, i=1, \cdots, n$.) It is the purpose of this paper to investigate the possibility of extending the homeomorphism $h$ so as to obtain a homeomorphism from $M$ onto $\mathfrak{M}$.

It will be shown that, if $M$ (and therefore $\mathfrak{M}$ ) is orientable, then $h$ cannot always be extended unless $n=1$. (A necessary and sufficient condition for the extendability is given in Theorem 1.) If $M$ (and therefore $\mathfrak{M}$ ) is non-orientable, then the extension is always possiblea result which, at first glance, may appear rather implausible.

These results are generalizations of the Schoenflies theorem [2, p. $324]^{2}$ and, astonishingly enough, do not appear to have been mentioned elsewhere. It is possible that they may serve as instruments in generalizing an extension theorem of Adkisson and MacLane [1] from a statement involving 2-spheres to one concerned with 2-manifolds. In any event, the theorems will be employed in the representation problem for Fréchet surfaces in a manner comparable to that by which a similar theorem was used to obtain a partial solution (Youngs [4]).

2. The theorems. Using the notation of the introduction, suppose that $M$ is orientable. A concordant orientation of $\left(J_{1}, \cdots, J_{n}\right)$ consists of an orientation on each Jordan curve, $J_{1}, \cdots, J_{n}$, such that the orientation induced on $M$ by the orientation on $J_{i}$ is independent of $i=1, \cdots, n$; or, in other words, there is an orientation on $M$ such that $J_{1}+\cdots+J_{n}\left(J_{i}\right.$ regarded as a cycle, $\left.i=1, \cdots, n\right)$ is the algebraic boundary of $M$. Hence each concordant orientation of $\left(J_{1}, \cdots, J_{n}\right)$ determines an orientation on $M$; namely, the orientation induced by $J_{i}$ for any $i=1, \cdots, n$. Conversely each orientation on $M$ determines a concordant orientation of $\left(J_{1}, \cdots, J_{n}\right)$; the orientation on $J_{i}$ being induced by the orientation on $M, i=1, \cdots, n$. 1947.

Presented to the Society, September 4, 1947; received by the editors September 4,

${ }^{1}$ Fellow of the John Simon Guggenheim Memorial Foundation.

2 Numbers in brackets refer to the bibliography. 
Thus there are two concordant orientations of $\left(J_{1}, \cdots, J_{n}\right)$; given one, the other is obtained by reversing the orientation on $J_{i}$, $i=1, \cdots, n$.

Now consider the homeomorphism $h$ and select a concordant orientation of $\left(J_{1}, \cdots, J_{n}\right)$. Then $J_{i}$ is oriented and $h \mid J_{i}$ (that is, $h$ restricted to $J_{i}$ ) determines an orientation on $\Im_{i}, i=1, \cdots, n$. This selection of orientations may or may not be a concordant orientation of $\left(\Im_{1}, \cdots, \Im_{n}\right)$. If it is, then $h$ is said to carry a concordant orientation of $\left(J_{1}, \cdots, J_{n}\right)$ into a concordant orientation of $\left(\Im_{1}, \cdots, \Im_{n}\right)$. It is obvious that if $h$ carries one of the two concordant orientations of $\left(J_{1}, \cdots, J_{n}\right)$ into a concordant orientation of $\left(\Im_{1}, \cdots, \Im_{n}\right)$, then it carries the other concordant orientation of $\left(J_{1}, \cdots, J_{n}\right)$ into the second concordant orientation of $\left(\Im_{1}, \cdots, \Im_{n}\right)$.

Now suppose that the homeomorphism $h$ can be extended so as to obtain a homeomorphism $h^{*}: M \rightarrow \mathfrak{M}$. (The heavy arrow indicates that the mapping is from $M$ onto $\mathfrak{M}$ ). Select an orientation on $M$ and consider the concordant orientation of $\left(J_{1}, \cdots, J_{n}\right)$ determined by the orientation on $M$. The homeomorphism $h \mid J_{i}$ induces an orientation on $\Im_{i}, i=1, \cdots, n$, while the homeomorphism $h^{*}$ induces an orientation on $\mathfrak{M}$. It follows that this orientation on $\mathfrak{M}$ induces an orientation on $\Im_{i}$ which is precisely that induced by $h \mid J_{i}, i=1, \cdots, n$. Consequently the orientation induced on $\Im_{i}$ by $h \mid J_{i}, i=1, \cdots, n$, yields a concordant orientation of $\left(\Im_{1}, \cdots, \Im_{n}\right)$. In other words, $h$ takes a concordant orientation of $\left(J_{1}, \cdots, J_{n}\right)$ into a concordant orientation of $\left(\Im_{1}, \cdots, \Im_{n}\right)$. Thus half of the first theorem listed below has been proved.

THEOREM 1. If $M$ and $\mathfrak{M}$ are homeomorphic orientable 2-manifolds with boundaries $B=J_{1} \cup \ldots \cup J_{n}$ and $\mathfrak{B}=\Im_{1}, \cup \ldots \cup \Im_{n}$ respectively $(n>0)$, then a homeomorphism $h: B \rightarrow \mathbb{B}$ can be extended to $a$ homeomorphism $h^{*}: M \rightarrow \mathfrak{M}$ if, and only if, $h$ carries a concordant orientation of $\left(J_{1}, \cdots, J_{n}\right)$ into a concordant orientation of $\left(\Im_{1}, \cdots, \Im_{n}\right)$.

THEOREM 2. If $M$ and $\mathfrak{M}$ are homeomorphic non-orientable 2-manifolds with boundaries $B=J_{1} \cup \ldots \cup J_{n}$ and $\mathfrak{B}=\Im_{1} \cup \ldots \cup \Im_{n}$ respectively $(n>0)$, then a homeomorphism $h: B \rightarrow \mathfrak{B}$ can always be extended to a homeomorphism $h^{*}: M \rightarrow \mathfrak{M}$.

Proof of THEOREM 1. The sufficiency of the condition needs to be established. Assuming that $h\left(J_{i}\right)=\Im_{i}, i=1, \cdots, n$, let $M^{*}$ and $\mathfrak{M}^{*}$ be the closed orientable 2-manifolds obtained by adjoining 2-cells to the bounding curves $J_{1}, \cdots, J_{n}$ and $\Im_{1}, \cdots, \Im_{n}$. These mani- 
folds are obviously homeomorphic; suppose that their 1-dimensional Betti number is $2 j, j \geqq 0$. By a suitable cutting of $M^{*}\left(\mathfrak{M}^{*}\right)$ one obtains the fundamental polygon $P^{*}: A A^{-1}\left(\mathfrak{B}^{*}: \mathfrak{A} \mathfrak{H}^{-1}\right)$, if $j=0$, or $P^{*}: A_{1} B_{1} A_{1}^{-1} B_{1}^{-1} \cdots A_{j} B_{j} A_{j}^{-1} B_{j}^{-1}\left(\mathfrak{B}^{*}: \mathfrak{U}_{1} \mathfrak{B}_{1} \mathfrak{H}_{1}^{-1} \mathfrak{B}_{1}^{-1} \cdots \mathfrak{A}_{j} \mathfrak{B}_{j} \mathfrak{A}_{j}^{-1} \mathfrak{B}_{j}^{-1}\right)$, if $j>0$, and the Jordan curves $J_{1}, \cdots, J_{n}\left(\Im_{1}, \cdots, \Im_{n}\right)$ are interior to $P^{*}\left(\mathfrak{P}^{*}\right)$. (See Seifert-Trelfall [3, chap. VI].) Let $J_{n+1}\left(\Im_{n+1}\right)$ be the Jordan curve boundary of $P^{*}\left(\mathfrak{P}^{*}\right)$ and $P^{\prime}\left(\mathfrak{P}^{\prime}\right)$ be the 2-manifold obtained from $P^{*}\left(\mathfrak{B}^{*}\right)$ by omitting the interiors of the 2-cells bounded by $J_{1}, \cdots, J_{n}\left(\Im_{1}, \cdots, \Im_{n}\right)$.

Select an orientation on $P^{\prime}$ and consider the induced orientations on $J_{1}, \cdots, J_{n+1}$. The mapping $h \mid J_{i}$ induces an orientation on $\Im_{i}$, $i=1, \cdots, n$. It follows from the hypothesis that the orientation on $\mathfrak{P}^{\prime}$ induced by $\Im_{i}$ is independent of $i=1, \cdots, n$. Consider $\Im_{n+1}$ to be given the orientation induced by the above orientation on $\mathfrak{B}^{\prime}$. It may be assumed that the order $\mathfrak{A} \mathfrak{A}-1$, if $j=0$, or $\mathfrak{A}_{1} \mathfrak{B}_{1} \mathfrak{A}_{1}^{-1} \mathfrak{B}_{1}^{-1} \ldots$ $\mathfrak{A}_{j} \mathfrak{B}_{j} \mathfrak{A}_{j}^{-1} \mathfrak{B}_{j}^{-1}$, if $j>0$, agrees with the orientation on $\Im_{n+1}$, and that the order $A A^{-1}$, if $j=0$, or $A_{1} B_{1} A_{1}^{-1} B_{1}^{-1} \cdots A_{j} B_{j} A_{j}^{-1} B_{j}^{-1}$, if $j>0$, agrees with the orientation on $J_{n+1}$.

If $j=0$ select the vertex $v(\mathfrak{b})$ which is the first point of $A$ (श्U). If $j>0$ select the vertex $v(\mathfrak{b})$ which is the first point of $A_{1}\left(\mathfrak{P}_{1}\right)$. Let $x_{i} \in J_{i}$ and $\mathfrak{x}_{i}=h\left(x_{i}\right) \in \Im_{i}, i=1, \cdots, n$. It follows that there are $\operatorname{arcs} Q_{1}, \cdots, Q_{n}$ $\left(\mathfrak{Q}_{1}, \cdots, \mathfrak{Q}_{n}\right)$ from $v$ to $x_{1}, \cdots, x_{n}\left(\mathfrak{v}\right.$ to $\left.\mathfrak{x}_{1}, \cdots, \mathfrak{x}_{n}\right)$ respectively, such that: $1^{\circ} . Q_{i} \cap Q_{k}=v\left(\mathfrak{Q}_{i} \cap \mathfrak{Q}_{k}=\mathfrak{v}\right), i \neq k ; i, k=1, \cdots, n$. $2^{\circ}$. If $P^{\prime}\left(\mathfrak{P}^{\prime}\right)$ is cut along these arcs then one obtains the polygon $P: Q_{1} J_{1} Q_{1}^{-1} \cdots Q_{n} J_{n} Q_{n}^{-1} A A^{-1}\left(\mathfrak{B}: \mathfrak{Q}_{1} \Im_{1} \mathfrak{Q}_{1}^{-1} \cdots \mathfrak{Q}_{n} \Im_{n} \mathfrak{Q}_{n}^{-1} \mathfrak{A} \mathfrak{A}^{-1}\right)$, if $j=0$, or $P: Q_{1} J_{1} Q_{1}^{-1} \cdots Q_{n} J_{n} Q_{n}^{-1} A_{1} B_{1} A_{1}^{-1} B_{1}^{-1} \cdots A_{j} B_{j} A_{j}^{-1} B_{j}^{-1} \quad(\mathfrak{B}$ $\left.: \mathfrak{Q}_{1} \mathfrak{\Im}_{1} \mathfrak{Q}_{1}^{-1} \ldots \mathfrak{Q}_{n} \Im_{n} \mathfrak{Q}_{n}^{-1} A_{1} \mathfrak{B}_{1} \mathfrak{P}_{1}^{-1} \mathfrak{B}_{1}^{-1} \ldots \mathfrak{A}_{j} \mathfrak{B}_{j} \mathfrak{A}_{j}^{-1} \mathfrak{B}_{j}^{-1}\right)$, if $j>0$. This is the fundamental polygon for $M(\mathfrak{M})$ and it is to be noted that the oriented boundary arcs $J_{1}, \cdots, J_{n}\left(\Im_{1}, \cdots, \Im_{n}\right)$ are found in the order of increasing indices in the above array. It follows that the homeomorphism $h$ carrying $J_{i}$ onto $\Im_{i}, i=1, \cdots, n$, can be extended to a homeomorphism $h$ from the boundary of $P$ onto the boundary of $\mathfrak{B}$ in such a manner that if $x$ and $y$ are to be identified by the identification mapping which obtains $M$ from $P$, then $h(x)$ and $h(y)$ are identified by the identification mapping which obtains $\mathfrak{M}$ from $\mathfrak{B}$.

Now by the Schoenflies theorem there is an extension $h^{*}$ of $h$ which maps $P$ homeomorphically onto $\mathfrak{P}$. The homeomorphism $h^{*}$ of the theorem is simply the above $h^{*}$ considered as a mapping from $M$ onto $\mathfrak{M}$.

Proof of Theorem 2. Assuming that $h\left(J_{i}\right)=\Im_{i}, i=1, \cdots, n$, let $M^{*}$ and $\mathfrak{M}^{*}$ be the closed non-orientable 2 -manifolds obtained by 
adjoining 2 -cells to the boundary curves $J_{1}, \cdots, J_{n}$ and $\Im_{1}, \cdots, \Im_{n}$. These manifolds are homeomorphic; suppose that their 1-dimensional Betti number is $(k-1), k>0$. By a suitable cutting of $M^{*}\left(\mathfrak{M}^{*}\right)$ one obtains the fundamental polygon $P^{*}: A_{1} A_{1} \cdots A_{k} A_{k}\left(\mathfrak{P}^{*}: \mathfrak{A}_{1} \mathfrak{A}_{1}\right.$ $\left.\cdots \mathfrak{A}_{k} \mathfrak{A}_{k}\right)$ and the Jordan curves $J_{1}, \cdots, J_{n}\left(\Im_{1}, \cdots, \Im_{n}\right)$ are interior to $P^{*}\left(\mathfrak{P}^{*}\right)$. (See Seifert-Trelfall [3, chap. VI].)

Consider a fixed orientation on $P^{*}$. This determines an orientation on $J_{i}$, and the homeomorphism $h \mid J_{i}$ induces an orientation on $\Im_{i}$, which, in turn, determines an orientation on $\mathfrak{B}^{*}, i=1, \cdots, n$. If this

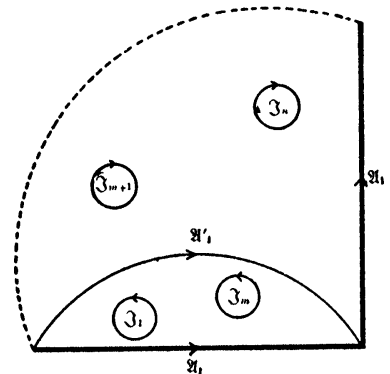

Fig. 1

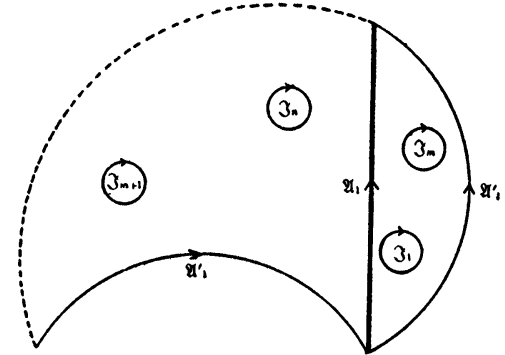

FIG. 2

orientation on $\mathfrak{B}^{*}$ is independent of $i=1, \cdots, n$, then it is readily seen that the proof can be completed as in Theorem 1. Suppose, therefore, that the orientations on $\Im_{1}, \cdots, \Im_{m}(m<n)$ determine one orientation on $\mathfrak{B}^{*}$ while those on $\Im_{m+1}, \cdots, \Im_{n}$ determine the other. There is a cross cut $\mathfrak{A}_{1}^{\prime}$ of $\mathfrak{B}^{*}$ joining the first point of $\mathfrak{A}_{1}$ to the last point of $\mathfrak{A}_{1}$ and separating $\Im_{1} \cup \ldots \cup \Im_{m}$ from $\Im_{m+1} \cup \ldots \cup \Im_{n}$. (See Fig. 1.) Cut $\mathfrak{B}^{*}$ along $\mathfrak{A}_{1}^{\prime}$ and identify the points of the two arcs labelled $\mathfrak{A}_{1}$ to obtain Fig. 2. Notice that in doing this one obtains the fundamental polygon $\mathfrak{P}^{\prime}: \mathfrak{A}_{1}^{\prime} \mathfrak{Y}_{1}^{\prime} \mathfrak{A}_{2} \mathfrak{A}_{2} \ldots \mathfrak{A}_{k} \mathfrak{A}_{k}$ of $\mathfrak{M}^{*}$ and $\mathfrak{Y}_{i}$ now determines an orientation on $\mathfrak{P}^{\prime}$ which is independent of $i=1, \cdots, n$. The proof is completed as in Theorem 1.

\section{BIBLIOGRAPHY}

1. V. W. Adkisson and Saunders MacLane, Extending maps of plane Peano continua, Duke Math. J. vol. 6 (1940) pp. 216-228.

2. A. Schoenflies, Betrage zur Theorie der Punktmengen. III, Math. Ann. vol. 62 (1906) pp. 286-328.

3. H. Seifert and W. Trelfall, Lehrbuch der Topologie, Leipzig, 1943.

4. J. W. T. Youngs, The topological theory of Fréchet surfaces, Ann. of Math. vol. 45 (1944) pp. 753-785.

INDIANA UNIVERSITY 